\title{
Impacto da terapia antirretroviral conforme diferentes consensos de tratamento da Aids no Brasil
}

\author{
Silvia Maria Gomes de Rossi, ${ }^{1}$ Eliane Cesario Pereira Maluf, ${ }^{2}$ \\ Denise Siqueira Carvalho, ${ }^{2}$ Clea Elisa Lopes Ribeiro ${ }^{3}$ \\ e Celia Regina Pissini Battaglin ${ }^{3}$
}

Sugestão de citação

Rossi SMG, Maluf ECP, Carvalho DS, Ribeiro CEL, Battaglin CRP. Impacto da terapia antirretroviral conforme diferentes consensos de tratamento da Aids no Brasil. Rev Panam Salud Publica. 2012;32(2):117-23.

RESUMO Objetivo. Comparar as características dos pacientes com Aids e os resultados dos tratamentos na vigência de três diferentes consensos de terapia antirretroviral preconizados pelo Ministério da Saúde do Brasil.

Métodos. Foram construídas coortes retrospectivas de pacientes com sobrevida de até 5 anos após diagnóstico. Os dados foram obtidos de prontuários, formulários de dispensação de medicamentos e declarações de óbitos dos pacientes residentes no município de Curitiba (PR), Brasil. Foram elegíveis 600 pacientes referentes aos 6 primeiros meses dos anos seguintes à implantação dos consensos (1992, 1997 e 2002).

Resultados. A razão de masculinidade passou de 6,5:1 em 1992 para 1,4:1 em 2002. Ocorreu um aumento proporcional na população com mais de 50 anos, que passou de 1,4\% em 1992 para 9,9\% em 2002. A letalidade diminuiu de 81,9\% para 33,9\% no período estudado. A análise dos que sobreviveram até pelo menos 5 anos após diagnóstico mostrou que a frequência de pacientes tratados aumentou, sendo 46,2, 94,0 e 91,7\%, respectivamente, para os anos de 1992, 1997 e 2002. A análise multivariada mostrou associação positiva e estatisticamente significante entre sobrevida até 5 anos após o diagnóstico de Aids e anos de estudo, faixa etária, ano de diagnóstico, tipo de terapia antirretroviral e adesão ao tratamento (todos com $\mathrm{P}<0,001$ ). Conclusões. O aprimoramento da terapia antirretroviral preconizada pelo Ministério da Saúde teve impacto positivo na sobrevida. Houve associação entre letalidade e menor escolaridade, maior faixa etária, diagnóstico obtido em 1992, tipo de terapia antirretroviral e adesão incompleta ao tratamento antirretroviral.

Palavras-chave Síndrome de imunodeficiência adquirida; sobrevida; letalidade; fármacos anti-Aids; Brasil.

1 Infectologia, Secretaria Estadual da Saúde do Paraná, Curitiba, Paraná, Brasil. Enviar correspondência para Silvia Maria Gomes de Rossi, silviaderossi@gmail.com

2 Saúde Comunitária, Universidade Federal do Paraná, Curitiba, Paraná, Brasil.

3 Centro de Epidemiologia, Secretaria Municipal da Saúde de Curitiba, Curitiba, Paraná, Brasil
Os avanços na terapia antirretroviral (TARV) levaram à diminuição da morbidade e da mortalidade por Aids, e estudos populacionais (1) mostram melhora na sobrevida dos pacientes com essa doença, havendo, aos 25 anos, uma expectativa mediana de vida de 32,5 anos desde o momento em que começam o tratamento.
Trabalho recente, com base em modelos prognósticos, com 4.174 pacientes holandeses portadores do HIV, avaliados no período de 1998 a 2007, estima que a expectativa média de vida de um paciente diagnosticado aos 25 anos, que ainda não teve doença oportunista e tem acesso ao tratamento, é de mais 52,4 anos. A expec- 
tativa média da população geral nesse mesmo modelo é de mais 53,1 anos (2).

Entre 1986 e 1997, a mediana de sobrevida era de 10,3 meses (3). Esse foi o período crítico da epidemia, pois se conhecia pouco sobre a Aids, e o manejo das infecções oportunistas era incipiente. Em 1986, a agência norte-americana reguladora de remédios e alimentos (Food and Drug Administration - FDA) aprovou a zidovudina para tratamento da Aids e, no Brasil, ela passou a ser fornecida pelo Ministério da Saúde (MS) em 1991. Nessa época, a TARV era baseada na monoterapia.

Outras drogas foram desenvolvidas, aumentando as opções de tratamento. Entre 1993 e 1994, surgiram os primeiros trabalhos sobre combinações de drogas, a TARV dupla (4), que passou a ser a TARV padrão no Brasil. A TARV dupla era indicada para pacientes com contagem de linfócitos TCD4 (CD4) entre 200 e 500 cels $/ \mathrm{mL}$, podendo ser utilizada a associação zidovudina + didanosina, zidovudina + zalcitabina ou zidovudina + lamivudina, incluindo o uso de um inibidor de protease (IP) se houvesse piora clínica ou imunológica, se fosse paciente grave virgem de TARV ou intolerante à zidovudina ou didanosina.

A TARV tríplice, mais eficaz, tornou-se padrão no mundo em 1996. Para determinar a proporção de pacientes que passaram a utilizar a TARV tríplice, foi constituída uma coorte com 2.267 adultos com Aids nos Estados Unidos, acompanhados entre janeiro de 1996 e janeiro de 1998, mostrando que a proporção de pacientes em uso dessa associação aumentou $70 \%$ (5). Outro trabalho, feito no Brasil com 3.930 adultos com Aids, mostrou sobrevida mediana de 18 meses para pacientes diagnosticados em 1995 e de 58 meses para aqueles que o foram em 1996; após ajustes estatísticos, concluiu-se que a melhora foi devida à TARV (6). O MS editou um novo consenso de TARV (7) em 2001 recomendando esquema antirretroviral tríplice em todos os casos que iniciariam tratamento, tendo CD4 $<350$ cels $/ \mathrm{mL}$, além dos sintomáticos.

Estudo realizado no Brasil analisou as taxas de mortalidade entre 1982 e 2002 e observou queda nessas taxas desde 1996, quando da introdução da TARV tríplice. Essa queda foi menor entre mulheres, adultos jovens e pessoas com menor nível socioeconômico, diminuindo a partir de 1999, provavelmente pela necessidade de diagnóstico mais precoce e acesso facilitado à assistência (8). Vários trabalhos mostram redução na hospitalização e na mortalidade por Aids, mas a procura tardia por diagnóstico e tratamento faz com que ainda ocorram casos somente diagnosticados após doença oportunista, aumentando o risco de transmissão e diminuindo a chance de resposta ao tratamento (9).

O MS brasileiro fornece atualmente medicação antirretroviral para aproximadamente 190.000 pacientes, sendo 21.000 no Paraná, na região Sul do país, e, destes, 10.000 estão em Curitiba, a capital do Estado. O Boletim Epidemiológico do ano de 2007, publicado pelo MS, apresenta dados de estudo feito com pacientes diagnosticados com Aids em 2002 e analisados 5 anos depois. O boletim revela que, até $2007,90 \%$ dos pacientes vivos estavam no Sudeste, $82 \%$ no Sul, $81 \%$ no Nordeste, $80 \%$ no Centro-Oeste e $78 \%$ no Norte. A média dos pacientes que foram a óbito até 1 ano após o diagnóstico foi de 6,1\% no Brasil como um todo (10).

Considerando-se a existência de poucos estudos comparando as respostas aos diferentes consensos, esta pesquisa teve como objetivo comparar as características dos pacientes com Aids e os resultados dos tratamentos na população maior de 13 anos de idade de Curitiba na vigência de três diferentes consensos de TARV, até 5 anos após o diagnóstico.

\section{MATERIAIS E MÉTODOS}

Trata-se de um estudo com três coortes retrospectivas. Foram incluídos todos os indivíduos com diagnóstico de Aids notificados ao Serviço de Epidemiologia nos primeiros 6 meses dos anos de 1992, 1997 e 2002, já que os consensos foram implantados em 1991, 1996 e 2001. Os pacientes eram de ambos os gêneros, acima de 13 anos de idade.

As informações foram coletadas em formulários específicos preenchidos a partir de consulta aos prontuários e complementadas junto ao Sistema de Informações de Mortalidade e formulários de dispensação de medicamentos, sem identificação dos pacientes. As variáveis constantes da ficha utilizada foram "ano do diagnóstico de Aids", "idade", "gênero", "anos de estudo", "categoria de exposição", "data do início do tratamento", "se houve adesão ao tratamento", "se a TARV utilizada foi monoterapia, terapia dupla ou tríplice", "se o paciente foi a óbito" e "data do óbito". Para avaliar a adesão, foram analisadas as anotações nos prontuários e nas fichas de dispensação dos medicamentos, sendo considerados aderentes os pacientes que compareciam às consultas agendadas e retiravam os medicamentos mensalmente.

Foram excluídos os casos com dados incompletos, os que foram a óbito por causas externas (assassinatos e suicídios) e os casos transferidos para outras localidades durante o estudo.

Foram considerados como sem tratamento os pacientes que nunca fizeram uso de antirretrovirais porque não havia indicação e aqueles em que o tempo de uso da medicação foi menor que 180 dias, considerado o prazo para que o vírus fique abaixo do limite de detecção de 50 cópias $/ \mathrm{mL}$, possibilitando maior recuperação imunológica e menor risco de resistência viral (11).

Os dados foram digitados em planilha do Excel e a análise foi realizada nos programas Epi-Info 6.4 e Stata versão 9. Após análise descritora das variáveis, avaliaram-se as possíveis associações com a chance de óbito por Aids. Inicialmente, foi realizada análise bivariada, aplicando-se o teste do qui-quadrado. Para fazerem parte da análise multivariada, foram selecionadas as variáveis que apresentaram um valor $p \leq 0,20$, método stepwise forward, e adotou-se o nível de significância de 0,05 para a interpretação dos resultados.

Este trabalho foi aprovado pelos Comitês de Ética do Hospital de Clínicas da Universidade Federal do Paraná e da Secretaria Municipal da Saúde de Curitiba, e teve a concordância da $2^{\text {a }}$ Regional de Saúde da Secretaria Estadual da Saúde do Paraná para a realização da pesquisa em suas dependências e acesso aos respectivos bancos de dados.

\section{RESULTADOS}

Neste estudo, foram selecionados inicialmente 708 casos, sendo 90 em 1992, 350 em 1997 e 268 em 2002. Deste número, foram excluídos 108 pacientes, 103 porque foram transferidos e perdeu-se a informação antes que fosse completado o tempo de estudo ou porque não se conseguiu obter sobre eles os dados necessários ao projeto, e 5 foram excluídos porque sua morte deveu-se a causas externas, sem relação com a Aids.

Em 1992, a taxa de letalidade foi de 81,9\%, diminuindo para 31,2\% em 1997 e para 33,9\% em 2002. 
O gênero masculino predominou em todos os períodos estudados, embora tenha diminuído ao longo do tempo, com a razão de masculinidade passando de 6,5:1, em 1992, para 1,4:1, em 2002.

Houve predomínio em todos os períodos da faixa etária entre 20 e 49 anos. No entanto, em 1997 e 2002, já se notou um aumento proporcional de casos na população com mais de 50 anos, que correspondia a 1,4\% do total dos casos em 1992, passando para 9,9\% em 2002.

Houve mudança quanto ao tipo de transmissão, diminuindo entre os homossexuais, entre os bissexuais e mesmo entre os usuários de drogas injetáveis (UDI), como se pode ver na figura 1. Já entre os heterossexuais, houve aumento na transmissão (de 33,9\%, em 1992, para 61,4\%, em 2002).

Quando foi analisada a escolaridade segundo o gênero, verificou-se que os homens apresentaram maior porcentual $(61,5 \%)$ entre os casos com maior escolaridade (de 8 a mais de 12 anos de estudo) em comparação com $48,5 \%$ das mulheres $(p<0,001)$, como mostra a tabela $1 . \mathrm{Na}$ análise específica do gênero masculino, constatou-se que a população homosse-

FIGURA 1. Distribuição proporcional dos casos de Aids referentes aos anos de 1992, 1997 e 2002, segundo a categoria de exposição ao HIV, Curitiba (PR), Brasil

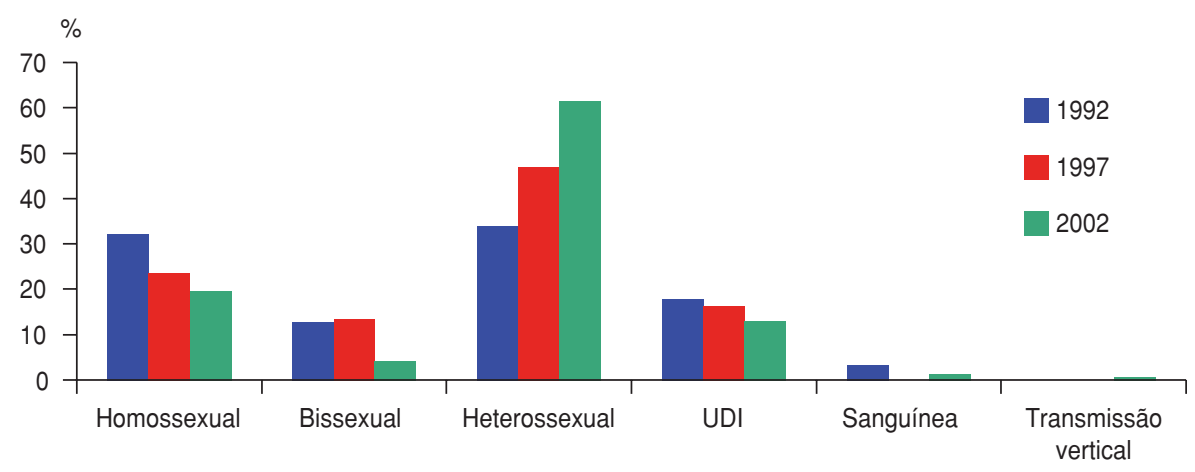

UDI: usuários de drogas injetáveis.

TABELA 1. Distribuição dos casos de Aids identificados no período estudado segundo anos de estudo e categoria de distribuição sexual, Curitiba (PR), Brasil

\begin{tabular}{|c|c|c|c|c|c|c|c|c|c|}
\hline \multirow{2}{*}{$\begin{array}{c}\text { Anos de } \\
\text { estudo }\end{array}$} & \multicolumn{3}{|c|}{ Feminino } & \multicolumn{3}{|c|}{ Masculino heterossexual } & \multicolumn{3}{|c|}{ Masculino homossexual } \\
\hline & No. & & $\%$ & No. & & $\%$ & No. & & $\%$ \\
\hline $0<4$ & 11 & & 6,7 & 3 & & 1,1 & 2 & & 1,9 \\
\hline $4<8$ & 73 & & 44,8 & 99 & & 37,4 & 15 & & 14,0 \\
\hline $8<11$ & 56 & & 34,4 & 83 & & 31,3 & 31 & & 28,9 \\
\hline 11 & 20 & $48,5^{a}$ & 12,3 & 56 & $61,5^{a}$ & 21,1 & 29 & $84,1^{a}$ & 27,1 \\
\hline$>12$ & 3 & & 1,8 & 24 & & 9,1 & 30 & & 28,1 \\
\hline Total & 163 & & 100,0 & 265 & & 100,0 & 107 & & 100,0 \\
\hline
\end{tabular}

Foram excluídos 65 casos com anos de estudos ignorados; período estudado: primeiro semestre dos anos de 1992, 1997 e 2002.

a $P<0,001$.
Em relação à regularidade no tratamento, em 1992 esta não influenciava a sobrevida, mas, em 1997 e 2002, a diferença entre o tratamento regular ou irregular era evidente $(65,6 \%$ e $58 \%$ versus $28,4 \%$ e $23,7 \%$ ).

Dos pacientes que sobreviveram até 5 anos após o diagnóstico, a maior parte fez tratamento, mesmo que de forma irregular, sendo $94 \%$ em 1997 e 91,7\% em 2002; já em 1992, o uso da medicação não levou à maior sobrevida, pois somente $46,2 \%$ dos que fizeram tratamento estavam vivos após 5 anos (tabela 5).

Os fatores avaliados, que estiveram relacionados ao óbito na análise multivariada foram menos de 4 anos de estudo, faixa etária maior que 50 anos, não realização de TARV e falta de adesão ao tratamento, e o ano de diagnóstico sendo 1992.

\section{DISCUSSÃO}

O tratamento do paciente com Aids tem sido um desafio. Conhecer os diferentes fatores que podem influenciar na resposta terapêutica é fundamental para reduzir a letalidade da doença.

No Brasil, especialmente na região Sudeste, entre 1986 e 1996, a ocorrência de Aids entre os homens aconteceu de forma independente da escolaridade; já entre as mulheres, houve aumento entre as de menor instrução (12). Estudo feito em São José (SC), no Sul do Brasil, apesar de não separar a análise por gênero, constatou que 50,6\% dos pacientes estudados completaram o $1^{\circ}$ grau, o que equivale a 8 anos de estudo, e que $15,3 \%$ não tinham nenhum grau de instrução (13)

Neste trabalho, os homens apresentaram maior escolaridade. Ao se agrupar o gênero masculino em heterossexuais e homossexuais, foi constatada que a maior escolaridade foi devida, em grande parte, aos homossexuais. Nos Estados Unidos, dos 2.898 pacientes notificados com Aids entre junho de 1990 e janeiro de 1993, somente $9 \%$ dos homossexuais brancos tinham menos de 12 anos de estudo (14). Analisando a influência da escolaridade sobre o tratamento, evidenciou-se, nesta pesquisa, que os pacientes com mais anos de estudo se trataram mais e com maior regularidade, talvez refletindo uma maior compreensão da importância e da necessidade do tratamento, assim como da adesão, devido ao risco de selecionar vírus HIV resistentes aos antirretrovirais em uso. 
TABELA 2. Distribuição dos casos de Aids identificados no período estudado segundo categoria de exposição e anos de estudo, Curitiba (PR), Brasil

\begin{tabular}{lcccccc}
\hline & \multicolumn{5}{c}{ Categoria de exposição ao HIV } \\
\cline { 2 - 7 } $\begin{array}{c}\text { Anos de } \\
\text { estudo }\end{array}$ & $\begin{array}{c}\text { Homossexual } \\
\text { (No.) }\end{array}$ & $\begin{array}{c}\text { Bissexual } \\
\text { (No.) }\end{array}$ & $\begin{array}{c}\text { Heterossexual } \\
\text { (No.) }\end{array}$ & $\begin{array}{c}\text { Usuário de drogas } \\
\text { injetáveis } \\
\text { (No.) }\end{array}$ & $\begin{array}{c}\text { Sanguínea } \\
\text { (No.) }\end{array}$ & $\begin{array}{c}\text { Transmissão } \\
\text { vertical } \\
\text { (No.) }\end{array}$ \\
\hline $0<4$ & 2 & 0 & 8 & 1 & 0 & 0 \\
$4<8$ & 15 & 12 & 90 & $36^{\mathrm{a}}$ & 4 & 1 \\
$8<11$ & 31 & 15 & 88 & 15 & 0 & 0 \\
11 & 29 & 16 & 39 & 8 & 0 & 0 \\
$>12$ & 30 & 6 & 12 & 1 & 0 & 0 \\
Total & 107 & 49 & 237 & 61 & 4 & 1 \\
\hline
\end{tabular}

Foram excluídos 65 casos com anos de estudos ignorados e 76 casos com categoria de exposição ignorada (sendo 18 casos com anos de estudo e categoria de exposição ignorados ao mesmo tempo); período estudado: primeiro semestre dos anos de 1992, 1997 e 2002.

a $P<0,001$.

TABELA 3. Distribuição dos casos de Aids no período estudado segundo anos de estudo e tratamento

\begin{tabular}{|c|c|c|c|c|c|c|}
\hline \multirow{3}{*}{$\begin{array}{l}\text { Anos de } \\
\text { estudo }\end{array}$} & \multicolumn{6}{|c|}{ Tratamento } \\
\hline & \multicolumn{2}{|c|}{ Não/nunca } & \multicolumn{2}{|c|}{ Sim/irregular } & \multicolumn{2}{|c|}{ Sim/regular } \\
\hline & No. & $\%$ & No. & $\%$ & No. & $\%$ \\
\hline $0<8$ & 70 & 53,8 & 78 & 49,1 & 57 & 22,7 \\
\hline$>8$ & 60 & 46,2 & 81 & 50,9 & 194 & $77,3^{a}$ \\
\hline Total & 130 & 100,0 & 159 & 100,0 & 251 & 100,0 \\
\hline
\end{tabular}

Foram excluídos 65 casos com anos de estudo ignorados; não/nunca: quem não tratou ou o fez por menos de 180 dias; período estudado: 1992, 1997 e 2002

a $P<0,001$.

TABELA 4. Distribuição dos casos de Aids no período estudado, segundo tipo de terapia antirretroviral (TARV) e evolução, Curitiba (PR), Brasil

\begin{tabular}{|c|c|c|c|c|c|}
\hline \multirow[b]{3}{*}{ TARV } & \multicolumn{5}{|c|}{ Evolução } \\
\hline & \multicolumn{2}{|c|}{ Vivos } & \multicolumn{2}{|c|}{ Mortos } & \multirow[b]{2}{*}{ Total } \\
\hline & No. & $\%$ & No. & $\%$ & \\
\hline Tríplice & $223^{a}$ & 66,5 & 47 & 38,2 & 270 \\
\hline Dupla + tríplice & 91 & 27,2 & 21 & 17,1 & 112 \\
\hline Mono + dupla + tríplice & 5 & 1,5 & 0 & 0 & 5 \\
\hline Mono + tríplice & 7 & 2,1 & 1 & 0,8 & 8 \\
\hline Dupla & 8 & 2,4 & 19 & 15,4 & 27 \\
\hline Mono + dupla & 1 & 0,3 & 6 & 4,9 & 7 \\
\hline Mono & 0 & 0 & 29 & 23,6 & 29 \\
\hline Total & 335 & 100,0 & 123 & 100,0 & 458 \\
\hline
\end{tabular}

Foram excluídos 142 pacientes que não usaram terapia antirretroviral; período estudado: primeiro semestre dos anos de 1992, 1997 e 2002.

a $P<0,001$.

Apresentaram maior chance de morrer os indivíduos com menor escolaridade, talvez por terem mais dificuldade de acesso aos serviços de saúde e, assim, ao diagnóstico precoce e à indicação mais precisa do tratamento específico, bem como maior dificuldade em compreender a importância desse tratamento. No Rio Grande do Norte, houve baixa adesão (caracterizada como interrupção do tratamento) após os pacientes terem alta hospitalar, entre usuários de drogas e aqueles com história de tratamento psi- quiátrico, baixo grau de escolaridade ou idade entre 25 e 34 anos (15). Trabalho que avaliou a sobrevida de pacientes com Aids realizado em Blumenau (SC) (16) mostrou, após análise multivariada, uma sobrevida menor entre os pacientes com menos anos de estudo, baixa contagem de CD4 e sem atendimento especializado. No Rio de Janeiro, em pesquisa com 945 pacientes, o abandono do tratamento teve associação negativa com nível de escolaridade mais alto, utilizando-se o modelo proporcional de Cox (17).
Os resultados da análise das características dos pacientes incluídos neste estudo mostraram que, já em 1997, começou a aumentar o número de casos diagnosticados em indivíduos com mais de 50 anos de idade. Essa constatação tem correspondência com os dados do Brasil, com 47.437 casos até junho de 2008 (9\% do total de casos), sendo $66 \%$ em homens (18). Esse fato é preocupante, pois se somam os fatores inerentes à senescência àqueles causados pelo HIV: uma inflamação crônica que gera mais rápido envelhecimento circulatório. Tal associação foi vista em trabalho prospectivo feito nos Estados Unidos com 122 pacientes e igual número de controles, mostrando que aqueles infectados pelo HIV tinham maior prevalência de hipertensão, hipertrigliceridemia, baixa densidade mineral óssea e lipodistrofia, além de fazerem mais uso de medicação antihipertensiva e hipolipemiante (19).

A faixa etária foi associada com maior chance de óbito, havendo maior risco naquela acima de 50 anos de idade (na análise bivariada, a odds ratio — OR foi de 1,77 com intervalo de confiança - IC de $95 \%$ entre 1,44 e 2,02), talvez por dificuldade de acesso ao diagnóstico precoce e pela maior incidência de comorbidades.

Os dados pesquisados mostraram que a sobrevida aumentou, com $18,1 \%$ dos casos diagnosticados em 1992 tendo sobrevivido 5 anos após o diagnóstico em Curitiba, enquanto isso aconteceu com 68,8\% e 66,1\% daqueles diagnosticados em 1997 e 2002, respectivamente. Isso provavelmente deve-se a uma melhora no tratamento, que, no princípio, era baseado em monoterapia sequencial, depois uma associação de dois medicamentos e, finalmente, passando para uma associação de três antirretrovirais, diminuindo, dessa forma, o risco de eclosão de vírus com mutações de resistência à TARV em uso.

Também se deve ressaltar que os pacientes que nunca receberam TARV $(24,6 \%)$ e sobreviveram provavelmente não apresentavam indicação clínica ou imunológica para o início do tratamento, mostrando provavelmente a importância do diagnóstico precoce. Na Espanha, foram estudados 610 pacientes internados entre janeiro de 1995 e dezembro de 2000, sendo analisados antes e depois da introdução da TARV tríplice, e este trabalho mostrou que a morbidade e a mortalidade diminuíram, aparecendo, no entanto, um aumento no número de internações $\mathrm{e}$ óbitos por doenças tradicionalmente não 
TABELA 5. Fatores relacionados ao óbito - análise multivariada

\begin{tabular}{lccccc}
\hline \multicolumn{1}{c}{ Característica } & No. & Mortos & $\%$ & OR (IC95\%) & $P$ \\
\hline Anos de estudo $<4^{a}$ & 535 & 186 & 34,8 & $1,57(1,23-2,01)$ & $<0,001$ \\
Faixa etária $>50$ anos & 600 & 230 & 38,3 & $1,44(1,13-1,83)$ & $<0,001$ \\
Ano do diagnóstico 1992 & 600 & 230 & 38,3 & $2,09(1,40-3,11)$ & $<0,001$ \\
$\begin{array}{l}\text { Terapia antirretroviral diferente da } \\
\text { tríplice }\end{array}$ & 600 & 222 & 37,0 & $1,16(1,04-1,28)$ & $=0,010$ \\
$\begin{array}{l}\text { Falta de adesão ao tratamento } \\
\quad \text { antirretroviral }\end{array}$ & 600 & 230 & 38,3 & $3,74(2,54-5,52)$ & $<0,001$ \\
\hline
\end{tabular}

${ }^{a}$ Foram excluídos 65 casos com anos de estudo ignorados; OR: odds ratio; IC $95 \%$ : intervalo de confiança de $95 \%$.

definidoras de Aids, como infecções respiratórias, doenças do aparelho digestivo e hepáticas (20). Ainda na Espanha, foram comparadas as respostas ao tratamento em duas épocas diferentes, logo após (1996) e bem depois (2001) da introdução da TARV tríplice, sendo encontrado que as diferenças de resposta ao tratamento entre os níveis de escolaridade baixo e alto diminuíram $70 \%$ em 2001, pelo efeito do tratamento mais eficaz (21).

Nesta investigação, constatou-se que os pacientes que receberam TARV tríplice trataram-se mais, tanto regular quanto irregularmente, do que aqueles que receberam outras formas de TARV $(p<0,001)$, havendo $66,5 \%$ deles vivos 5 anos após o diagnóstico. Esse resultado reflete sua maior eficácia e, talvez, uma melhor indicação de seu uso, com um diagnóstico mais precoce, antes da imunidade estar muito comprometida - fatores que influenciam na adesão ao tratamento. Ressalta-se que a falta de adesão à TARV tríplice está associada ao aumento da mortalidade (22). O impacto de tratamentos anteriores foi estudado em 3.538 pacientes da Califórnia, Estados Unidos, sendo visto que ser virgem de tratamentos anteriores teve efeito positivo na adesão e na melhora tanto no número de células CD4 quanto nos níveis de viremia, provavelmente pela não seleção de vírus HIV resistentes, o que é mais comum em tratamentos menos eficazes (23). Em São Paulo, 90\% dos pacientes vivos após 10 anos estavam usando TARV combinada ( $82 \%$ com 3 ou mais drogas, e $8 \%$ com 2 ), e $10 \%$ continuavam sem medicação (24).

Neste estudo, a maior parte dos que sobreviveram mais do que 5 anos após o diagnóstico fez tratamento, e este, mesmo irregular, teve reflexos no aumento da sobrevida, ao contrário de quem não tratou ou o fez por muito pouco tempo para atingir algum resultado. Esse dado reflete a importância da indicação no momento adequado e do uso da melhor TARV disponível, para se obter maior sobrevida.
Em 1997, 65,6\% e, em 2002, 58\% dos pacientes fizeram tratamento regular e estavam vivos 5 anos após o diagnóstico, enquanto dos que fizeram tratamento irregular somente $28,4 \%$, em 1997, e 23,7\%, em 2002, permaneceram vivos. Em 1992 não houve essa diferença porque o uso de monoterapia (na época era disponível somente a zidovudina) não tinha maiores reflexos no aumento da sobrevida, principalmente após 5 anos de diagnóstico; também em relação a esse ano, verificou-se que os pacientes não apresentavam diferença na relação da adesão com a escolaridade, provavelmente porque, nessa época, o tratamento, baseado na monoterapia, não sofria impacto da adesão, como mostra trabalho realizado com 1.965 pacientes com Aids nos Estados Unidos: o tempo entre diagnóstico e óbito aumentou de 0,56 anos (IC95\%: 0,50-0,64) no tempo da monoterapia para 5,08 anos (IC95\%: 2,39-10,79) já na era da TARV tríplice (25). Estudo também realizado nos Estados Unidos mostrou que o risco de infecções oportunistas diminuiu $81 \%$ quando se comparam as eras da monoterapia e TARV tríplice (26).

Neste trabalho, constata-se que 91,4\% dos pacientes que iniciaram tratamento em 2002 o fizeram com esquema contendo três drogas, refletindo as orientações do consenso nacional. Evidencia-se ainda que, entre os pacientes que não receberam tratamento, a maior parte foi a óbito até o $5^{\circ}$ ano após o diagnóstico de Aids, assim como os achados de estudo feito com 536 homens com data de infecção pelo HIV conhecida, nos Estados Unidos, e acompanhados por 13 anos, que evidenciou maior tempo para desenvolver Aids e maior sobrevida, além de interrupção da queda das células CD4 após a introdução da TARV tríplice (27).

Avaliando a adesão ao tratamento com relação à categoria de exposição, nota-se que somente uma terça parte dos UDIs fizeram tratamento regular, em comparação com os demais grupos. Esse valor é semelhante ao de um trabalho realizado no Brasil (28) comparando o acesso e a resposta ao tratamento entre UDI e homossexuais masculinos, apontando maior risco de morte entre os UDIs (OR: 1,94, IC95\%: 1,84-2,05). Os UDIs, comparados com os homossexuais masculinos, chegam a ter $33 \%$ menos oportunidade de iniciar tratamento, menor supressão da carga viral $(\mathrm{CV})$ e mortalidade 2,5 vezes maior, segundo dados coletados na Espanha entre 4.643 pacientes (29). Nos Estados Unidos, a incidência relativa de doenças definidoras de Aids entre UDI em tratamento foi maior que entre os não usuários de drogas, sugerindo dificuldades no uso correto da TARV entre os UDIs (30).

Tem-se presente que a adesão à TARV, como em qualquer tratamento que envolva antimicrobianos, é extremamente importante, pois, se as concentrações destes diminuem, os micro-organismos conseguem se multiplicar. Entre os que conseguem, alguns podem ter sofrido uma mutação que confira resistência aos medicamentos em uso. Apesar de dificilmente os índices de adesão chegarem aos $100 \%$, sabe-se que, quanto mais altos, menor o risco de mutantes resistentes serem selecionados (31). A concentração sérica do HIV diminui em aproximadamente $99 \%$ nas duas primeiras semanas após o início de terapia eficaz, seguindo um padrão bifásico, em que, na segunda fase, a CV diminui gradativamente (32). Uma CV acima do nível de detecção após 6 meses de tratamento é geralmente considerada falência terapêutica.

A primeira limitação no presente trabalho foi ser uma pesquisa retrospectiva, com dados secundários e a consequente dificuldade em coletá-los. Não foi possível obter todas as informações de 103 casos, apesar do esforço em tentar averiguar se, entre eles, ocorreu algum óbito no período estudado fora de Curitiba. A segunda limitação veio da comparação entre diferentes períodos, considerandose que houve avanços tecnológicos que influenciaram a indicação mais precisa da TARV.

Esta pesquisa mostrou que o aprimoramento da TARV preconizada pelo MS do Brasil teve impacto positivo na sobrevida dos pacientes estudados, principalmente levando-se em conta a escolaridade, a faixa etária, o ano de diagnóstico da Aids, o tipo de TARV e a adesão ao tratamento. Não foi evidenciada associação entre sobrevida, gênero e categoria de exposição. 
A importância de se identificarem fatores que reduzem a sobrevida do paciente com Aids está em contribuir no planejamento das ações de redução da morbimortalidade da doença pois, de posse dessas informações, poderão ser elaboradas estratégias e efetuados investimentos direcionados a pontos específicos, como a importância do diagnóstico precoce, da necessidade da Aids ser pensada como diagnóstico diferencial de várias enfermidades, e da observação atenta de possíveis alterações nas categorias de transmissão para um melhor direcionamento das medidas preventivas, além das atualizações com relação à melhor terapêutica disponível e melhor adesão ao tratamento. Mostraram-se ainda as vantagens na existência de consensos de tratamento, pois otimizam os custos dentro da lógica do maior benefício aos pacientes, além de reduzir a resistência a medicamentos decorrente de seu uso indiscriminado.

\section{REFERÊNCIAS}

1. Lohse N, Hansen AB, Pedersen G, Kronborg G, Gerstoft J, Sørensen HT, et al. Survival of persons with and without HIV infection in Denmark, 1995-2005. Ann Intern Med. 2007;146(2):87-95.

2. van Sighem AI, Gras LA, Reiss P, Brinkman $\mathrm{K}$, de Wolf $\mathrm{F}$; ATHENA national observational cohort study. Life expectancy of recently diagnosed asymptomatic HIV-infected patients approaches that of uninfected individuals. AIDS. 2010;24(10):1527-35.

3. Menesia EO, Passos AD, Monteiro ME, Dal-Fabbro AL, Laprega MR. Sobrevivência de pacientes com aids em uma cidade do sudeste brasileiro. Rev Panam Salud Publica. 2001;10(1):29-36.

4. Estados Unidos da América, National Institute of Allergy and Infectious Diseases. Bethesda, MD: ACTG 106: AZT/ddc combination shows benefits for patients with advanced HIV disease. Disponível em: http://aids info.nih.gov/news/42/actg-106-azt-ddccombination-shows-benefits-for-patientswith-advanced-hiv-disease/ Acessado em 22 de junho de 2008.

5. Cunningham WE, Markson LE, Andersen RM, Crystal SH, Fleishman JA, Golin C, et al. Prevalence and predictors of highly active antiretroviral therapy use in patients with HIV infection in the United States. J Acquir Immune Defic Syndr. 2000;25(2):115-23.

6. Marins JRP, Jamal LF, Chen SY, Barros MB, Hudes ES, Barbosa AA, et al. Dramatic improvement in survival among Brazilian AIDS patients. AIDS. 2003;17(11):1675-82.

7. Brasil. Ministério da Saúde. Recomendações para terapia anti-retroviral em adultos e adolescentes infectados pelo hiv - 2001. Brasília, DF. Disponível em: http://www.aids. gov.br/sites/default/files/Consenso_ARV_ adulto_-_versao_2001.pdf Acessado em 20 de novembro de 2006.

8. Reis AC, Santos EM, Cruz MM. A mortalidade por aids no Brasil: um estudo exploratório de sua evolução temporal. Epidemiol Serv Saúde. 2007;16(3):193-205.

9. Keruly JC, Moore RD. Immune status at presentation to care did not improve among antiretroviral-naïve persons from 1990 to 2006. Clin Infect Dis. 2007;45(10):1369-74.

10. Brasil. Ministério da Saúde. Boletim Epidemiológico 2007. Brasília, DF. Disponível em: http://www.aids.gov.br/sites/default/ files/Boletim2007_internet090108.pdf Acessado em 22 de novembro de 2007.
11. Smith CJ, Staszewski S, Sabin C, Nelson M, Dauer B, Gute P, et al. Use of viral load measured after 4 weeks of highly active antiretroviral therapy to predict virologic outcome at 24 weeks for HIV-1 positive individuals. J Acquir Immune Defic Syndr. 2004;37(1): 1155-9.

12. Fonseca MG, Bastos FI, Derrico M, Andrade CLT, Travassos C, Szwarcwald CL. Aids e grau de escolaridade no Brasil: evolução temporal de 1986 a 1996. Cad Saude Publica. 2000;16(Supl 1):77-87.

13. Bertoni RF, Bunn K, Silva J, Tracbert J. Perfil demográfico e socioeconômico dos portadores de HIV/AIDS do ambulatório de controle de DST? AIDS de São José, SC. Arq Catarin Med. 2011;4(39):75-9.

14. Diaz T, Chu SY, Buehler JW, Boyd D, Checko PJ, Conti L, et al. Socioeconomic differences among people with aids: results from a multistate surveillance Project. Am J Prev Med. 1994;10(4):217-22.

15. Brito AM, Szwarcwald CL, Castilho E. Fatores associados à interrupção de tratamento antirretroviral em adultos com aids. Rio Grande do Norte, Brasil, 1999-2002. Rev Assoc Med Bras. 2006;52(2):86-92.

16. Santa Helena ET, Mafra ML, Simes M. Fatores associados à sobreviva de pessoas vivendo com aids no Município de Blumenau, Estado de Santa Catarina, Brasil, 1997-2004. Epidemiol Serv Saúde. 2009;18(1):45-53.

17. Schilkowsky LB, Portela MC, Sá MC. Fatores associados ao abandono de acompanhamento ambulatorial em um serviço de assistência especializada em HIV/aids na cidade do Rio de Janeiro, RJ. Rev Bras Epidemiol. 2011;14(2): $187-97$.

18. Brasil. Ministério da Saúde. Boletim Epidemiológico 2008. Brasília, DF. Disponível em: http://www.aids.gov.br/sites/default/ files/Boletim2008_versao1_6.pdf Acessado em 10 de dezembro de 2011.

19. Onem NF, Overton ET, Seyfried W, Stumm ER, Snell M, Mondy K, et al. Aging and HIV infection: a comparison between older HIVinfected persons and the general population. HIV Clin Trials. 2010;11(2):100-9.

20. Escolano Hortelano CM, Ramos Rincón JM, Gutiérrez Rodero F, Masiá Canuto M, Hernández Aguado I, Benito Santaleocadia C, et al. Cambios en el espectro de la morbilidad y la mortalidad de los ingresos hospitalarios de los pacientes con infección por el virus de la inmunodeficiencia humana durante la era del tratamiento antirretroviral de gran actividad. Med Clin-Barcelona. 2004;122(1):1-5.

21. Sánchez E, Regidor E, Fuente L, Luquero FJ, Mateo S. Papel del tratamiento antiretroviral en la reducción de la asociación entre nivel educativo bajo y mortalidad por sida. Med Clin-Barcelona. 2008;130(4):133-5.

22. Lima VD, Harrigan R, Bangsberg DR, Hogg RS, Gross R, Yip B, et al. The combined effect of modern highly active antiretroviral therapy regimens and adherence on mortality over time. J Acquir Immune Defic Syndr. 2009;50(5):529-36.

23. Horberg M, Silverberg M, Hurley L, Delorenze $\mathrm{G}$, Quesenberry C. Influence of prior antiretroviral experience on adherence and responses to new highly active antiretroviral therapy regimens. AIDS Patient Care STDS. 2008;22(4):301-12.

24. Casseb J, Fonseca LA, Veiga AP, Almeida A, Bueno A, Ferez AC, et al. Aids incidence and mortality on a hospital-based cohort of HIV-1 seropositive patients receiving highly active antiretroviral therapy in São Paulo, Brazil. AIDS Patient Care STDS. 2003;17(9):447-52.

25. Schneider MF, Gange SJ, Williams CM, Anastos K, Greenblatt RM, Kingsley L, et al. Patterns of the hazard of death after AIDS through the evolution of antiretroviral therapy: 1684-2004. AIDS. 2005;19(17):2009-18.

26. Detels R, Tarwater P, Phair JP, Margolick J, Riddler SA, Muñoz A; Multicenter AIDS Cohort Study. Effectiveness of potent antiretroviral therapies on the incidence of opportunistic infections before and after AIDS diagnosis. AIDS. 2001;15(3):347-55.

27. Detels R, Muñoz A, McFarlane G, Kingsley LA, Margolick JB, Giorgi J, et al. Effectiveness of potent antiretroviral therapy on time to aids and death in men with known HIV infection duration. JAMA. 1998;280(17):1497-503.

28. Malta M, Bastos FI, Silva CMFP, Pereira GFM, Lucena FFA, Fonseca MGP, et al. Differential survival benefit of Universal HAART Access in Brazil: a Nation-Wide comparison of injecting drug users versus men who have sex with men. J Acquir Immune Defic Syndr. 2009;52(5):629-35.

29. Rodríguez-Arenas MA, Jarrín I, del Amo J, Iribarren JA, Moreno S, Viciana P, Peña A, Sirvent JL, Vidal F, Lacruz J, Gutierrez F, Oteo JA, Asencio R, Castilla J, Hoyos SP; CoRIS-MD. Delay in the initiation of HAART, poorer virological response, and 
higher mortality among HIV-infected injecting drug users in Spain. AIDS Res Hum Retrov. 2006;22(8):715-23.

30. Moore RD, Keruly JC, Chaisson RE. Differences in HIV disease progression by injecting drug use in HIV-infected persons in care. J Acquir Immune Defic Syndr. 2004;35(1): $46-51$.
31. Lima VD, Harrigan R, Murray M, Moore DM, Wood E, Hogg RS, et al. Differential impact of adherence on long-term treatment response among naïve HIV-infected individuals. AIDS. 2008;22(17):2371-80.

32. $\mathrm{Wu} \mathrm{H}$, Kuritzkes DR, McClernon DR, Kessler $\mathrm{H}$, Connick E, Landay A, et al. Characterization of viral dynamics in HIV type 1 infected patients treated with combination antiretroviral therapy: relationships to host factors, cellular restoration, and virologic end points. J Infect Dis. 1999;179:799-807.

Manuscrito recebido em 17 de setembro de 2011. Aceito em versão revisada em 10 de junho de 2012.

ABSTRACT Objective. Compare the characteristics of AIDS patients and treatment outcomes under three different antiretroviral treatment regimens advocated by the Ministry of Health of Brazil.

Impact of antiretroviral therapy under different treatment regimens

Methods. Retrospective cohorts of patients who had survived up to five years after diagnosis were constructed. The data were obtained from medical records, medication dispensing forms, and death certificates of patients in Curitiba, in the Brazilian state of Paraná. Six hundred patients were selected from the first six months following the adoption of each of the treatment regimens (1992, 1997, and 2002).

Results. The ratio of men to women fell from 6.5:1 in 1992 to 1.4:1 in 2002. There was a proportionate rise in the number of people over 50 , which increased from $1.4 \%$ in 1992 to $9.9 \%$ in 2002 . The case fatality rate dropped from $81.9 \%$ to $33.9 \%$ in the period in question. An analysis of those who survived at least five years from the date of diagnosis showed that the percentage of patients treated increased from $46.2 \%$ in 1992 to $94.0 \%$ in 1997, finishing at $91.7 \%$ in 2002. Multivariate analysis yielded a positive and statistically significant association between survival up to five years after an AIDS diagnosis and years of schooling, age group, year of diagnosis, type of antiretroviral therapy, and treatment adherence (all with $P<0.001$ ).

Conclusions. Continuous improvement of the antiretroviral therapy recommended by the Ministry of Health had a positive impact on survival. There was an association between case fatality and fewer years of schooling, membership in an older age group, a diagnosis obtained in 1992, the type of antiretroviral therapy, and suboptimal adherence to antiretroviral treatment regimens.

Key words Acquired immunodeficiency syndrome; survivorship (public health); lethality; antiHIV agents; Brazil. 